\title{
Precedence permutation patterns creating criticality constellations: Exploring a conjecture on nonlinear activities with continuous links
}

DOI 10.1515/otmcj-2016-0023

Received August 31, 2017; accepted November 23, 2017

\begin{abstract}
The inaugural challenge of the 2016 Creative Construction Conference has posed two related questions on how many possible criticality constellations with different behaviors for delays and acceleration exist and how said constellations can occur for nonlinearly and monotonously progressing activities that have continuous relations. This paper systematically solves these questions by performing a thorough literature review, assembling theoretical foundations for link constellations, performing a computer simulation of all possible permutations, and providing a mathematical proof by contradiction. It is found that (for the initially assumed self-contained activities in a network schedule that exhibit only a linearly growing production), three newly hypothesized criticality constellations cannot exist. Nonlinear activity constellations with diverging or converging relative productivities are examined next. Lags in networks become buffers in linear schedules. It is found that a nonlinear curvature of the progress may induce middle-to-middle relations besides those between start and finish. If multiple curvatures are allowed, then partial segments can form relations, which increase the number of criticality constellations. This paper is extended from the 2017 Procedia Engineering conference version.
\end{abstract}

Keywords: precedence diagramming, link constellations, continuous precedence relations, classification of critical activities

\footnotetext{
*Corresponding author: Gunnar Lucko, Catholic University of America Washington, DC UNITED STATES, E-mail: lucko@cua.edu Yi Su, Postdoctoral Research Associate Department of Civil Engineering Catholic University of America, E-mail: 61su@cua.edu
}

\section{Introduction}

The theory of network schedules in construction project management is celebrating its 60th anniversary after Kelley and Walker (1959) conceived the widely known critical path method around December 1956 (Kelley and Walker 1989) for schedules that are represented as networks. It constitutes a simplified form of linear programming with a rigorous equation structure of start plus duration equals finish $(\mathrm{S}+\mathrm{D}=\mathrm{F})$ for all activities. It has two "passes": first, adding all activity durations sequentially from the origin and taking the maximum at any merge of several predecessors under an "as-early-as-possible" assumption (forward pass, which also gives the project duration) and second, subtracting all activity durations sequentially from the terminus and taking the minimum at any merge of several successors under an "as-late-as-possible" assumption (backward pass). If links carry lead or lag durations, they are added or subtracted analogously. Comparing the earliest and latest start and finish points from these passes determines the flexibility (float) of activities to be delayed without harm. Like activities and leads or lags, float is measured in time units, commonly workdays. If it is zero, the activity is deemed critical. This means that its delay will immediately impact the project duration. Of interest for this paper is how the link types in the sequence impact the possible criticality of an activity.

\section{Literature review}

Recent papers by Hajdu (e.g., 2015b) have renewed a focus on the development and theory of the precedence diagramming method by Fondahl (1962) as expanded by IBM (1964) to four different link types, as opposed to the default finish-to-start link that had limited the realism of the critical path method of Kelley and Walker (1959). Continuous relations between two activities with repetitive 
tasks were shown as a multitude of task links (Hajdu 2015b). The transition from end-point links to true continuous relations between activity pairs was demonstrated for monotonous and invertible functions to model nonlinear progress of activities (Hajdu 2015a), e.g., increasing or decreasing productivities that are caused by a changing numerator or denominator, e.g., learning or fatigue, or a changing geometry of the work product itself, e.g., a deepening trench.

Theoretical studies of network scheduling have punctuated its history, which this paper can only review briefly. Roy (1959) named the metra potential method after his consulting firm. Unknown to Fondahl (1962), it pioneered activity-on-node diagrams instead of activity-on-arrow diagrams by Kelley and Walker (1959) and enabled links between activity starts. Confusingly, the name of nontime-scaled network diagrams has become intermingled with another technique, the program evaluation and review technique (Fazar 1962), which introduced a threepoint estimate of probabilistic durations. Interestingly, the literature continued to mention the complete four different link types (see Section 2.1) only after scheduling calculations were explained (Harris 1978), which fittingly echoed their historically "later" addition to theory.

Besides describing antecedents of linear schedules, which have been reviewed by Lucko and Gattei (2016), Rösch (1970) compared the methods of Roy (1959) and Fondahl (1962) with small examples in bar chart, network schedule, and linear schedule representations, including some older variants. Wiest (1981) made a seminal contribution by describing how activity pairs can be linked in a manner that impacts the project duration in a normal, reverse, neutral, or perverse manner. Kallantzis and Lambropoulos (2004) showed how such a reverse behavior is explained with a linear schedule.

Hajdu (2015c), in a review of precedence diagramming for which this author provided sources from the literature, highlighted studies that recently have sought to generalize end-point links to newly being able to attach anytime during mid-activity: The chronographic method (e.g., Francis 2004) and graphical diagramming method (Ponce de Leon 2008) for time-scaled precedence diagrams (Harris 1978) (called line schedules for short), which unlike bar charts, hold multiple activities per row and may have evolved from the arrow diagramming method; the "beeline" diagram (Kim 2012) that also allows multiple links between point pairs on two activities; and the relationship diagramming method (Plotnick 2006), whose single link carries some explanatory codes. Yet the assumptions of these methods are beyond the scope of this paper as are maximum lags as links constraints (Hajdu (1997), which may act jointly with minimum ones or act alone. Mubarak (2015) described a "dynamic" but ultimately limited concept of minimum lag, whose predecessor can have a flexible shape. More pertinent to this paper is the generalized case of continuous precedence relations that Hajdu et al. (2017) have written with singularity functions, which can model nonlinearity in both the activity progress and buffers between them.

\subsection{Network schedule assumptions}

Assumptions for this paper have been set by the challenge (Hajdu 2016; Hajdu et al. 2016) as representing schedules as activity-on-node networks, i.e., acyclic (no loops) graphs, which are directional from an origin to a terminus node, whose activities are connected at their ends via discrete end-point links and progress at a constant productivity without interruptibility.

Assuming that two activities are connected via only one link and that it may attach at either the start or finish of a predecessor and successor, $2 \times 2=4$ possible one-link relations can exist: finish to start, start to start, finish to finish, and start to finish. Broadening this common list to connect the four start and finish points of the activity pair with more links gives two-link, three-link, and fourlink end-point relations (the upper limit) as follows. Each link can carry a lead or lag, which means either a period seen from predecessor or successor view (Crandall 1973) or a negative or positive link duration (Mubarak 2015). Together, 15 different permutations of connecting the activity pair can exist as follows:

- one-link permutations: $\{\mathrm{FS}\},\{\mathrm{SS}\},\{\mathrm{FF}\}$, and $\{\mathrm{SF}\}$

- two-link permutations: $\{\mathrm{FS}, \mathrm{SS}\},\{\mathrm{FS}, \mathrm{FF}\},\{\mathrm{FS}, \mathrm{SF}\}$, $\{\mathrm{SS}, \mathrm{FF}\},\{\mathrm{SS}, \mathrm{SF}\}$, and $\{\mathrm{FF}, \mathrm{SF}\}$

- three-link permutations: $\{\mathrm{FS}, \mathrm{SS}, \mathrm{FF}\},\{\mathrm{FS}, \mathrm{SS}, \mathrm{SF}\}$, $\{\mathrm{FS}, \mathrm{FF}, \mathrm{SF}\}$, and $\{\mathrm{SS}, \mathrm{FF}, \mathrm{SF}\}$

- four-link permutations: $\{\mathrm{FS}, \mathrm{SS}, \mathrm{FF}, \mathrm{SF}\}$

\subsection{Challenges of criticality constellations}

Following a listing of the four one-link end-point relations, the inaugural challenge of the 2016 Creative Construction Conference (Hajdu 2016) has classified the criticality constellations by whether an increase (i.e., delay) or decrease (i.e. acceleration) in the activity duration will cause the project duration to increase, decrease, or not be impacted $(+,-$, or 0 , respectively). The challenge illustrated this phenomenon with influence lines, which have roots in early scheduling literature (Mauchly 1962). 
Tab. 1: Criticality configurations with impact of activity change on project duration (after Hajdu 1997).

\begin{tabular}{|c|c|c|c|c|c|c|c|}
\hline Activity & Project & Activity & Project & Incoming & Outgoing & Description & Name \\
\hline+ & + & - & - & FS & FS & Normal-normal & +- \\
\hline+ & - & - & + & $\mathrm{FF}$ & SS & Reverse-reverse & -+ \\
\hline \multirow[t]{2}{*}{+} & 0 & - & 0 & FS & SS & Start-neutral & 00 \\
\hline & & & & $\mathrm{FF}$ & FS & Finish-neutral & \\
\hline \multirow[t]{2}{*}{+} & + & - & 0 & FS & SS, FS & Normal-neutral (in) & +0 \\
\hline & & & & $\mathrm{FS}, \mathrm{FF}$ & FS & Normal-neutral (out) & \\
\hline \multirow[t]{2}{*}{+} & 0 & - & + & $\mathrm{FF}$ & SS, FS & Neutral-reverse (in) & $0+$ \\
\hline & & & & $\mathrm{FS}, \mathrm{FF}$ & SS & Neutral-reverse (out) & \\
\hline+ & + & - & + & $\mathrm{FS}, \mathrm{FF}$ & SS, FS & Normal-reverse "perverse" & ++ \\
\hline+ & - & - & 0 & Unknown & Unknown & Reverse-neutral & -0 \\
\hline+ & 0 & - & - & Unknown & Unknown & Neutral-normal & $0-$ \\
\hline+ & - & - & - & Unknown & Unknown & Reverse-normal & -- \\
\hline
\end{tabular}

Table 1 lists three newly hypothesized constellations that have been derived by creating $3 \times 3=9$ permutations of possible impacts on the project duration from shorter activities and longer activities (Hajdu 1997, expanded from Bokor and Hajdu 2015). It makes the implicit assumptions that a change in duration will result in a respective movement of a "free" activity end and that only end-point links with discrete lags (not continuous relations with buffers like in linear schedules) exist.

Despite the longstanding and rich research in the area of construction scheduling, surprisingly such an interaction of link structures and the criticality that they can potentially generate (as Table 1 summarizes) have not been researched exhaustively. Therefore, a conjecture has been created (Hajdu 2016, p. 12), which poses the challenge that this research explores:

- The number of critical activity types does not change if ... non-linear strictly increasing continuous activity production-time function, ... point-to-point relations ... and continuous relations are allowed in the network.

The subsequent paper (Hajdu et al. 2016, p. 10) rephrased this conjecture somewhat:

- Critical activities of the generalized PDM network described in ... this paper can be classified into the same six classes as critical activities of the traditional PDM model with the four end-point relations.

This conjecture is addressed through three research questions that the research methodology will investigate in turn:

- Research question 1: How many criticality constellations with different behaviors due to changes in duration exist in network schedules with linearly progressing activities that are connected with discrete end-point link relations?
- Research question 2: How can said criticality constellations occur for nonlinearly and monotonously progressing activities that are connected with continuous relations? This question will be examined by using linear schedules.

- Research question 3: How can influence lines, which visualize how the duration of a single activity influences the total project duration, be generalized to constellations of multiple activities?

\subsection{Research goal and objectives}

The goal of this research is to understand the possible varieties of criticality that arise from different relations between activities in network schedules and linear schedules, which are addressed via three research objectives:

- Research objective 1: identify and prove mathematically possible number of different criticality constellations.

- Research objective 2: investigate existence of possible criticality constellations that include nonlinear activities.

- Research objective 3: generalize influence lines toward including predecessors and successors of activities.

\section{Methodology}

The methodology uses a theory-building approach that enumerates and simulates all possible permutations of links between activities to gain the understanding to formulate the desired proof, illustrated by network and linear schedule constellations. A pertinent selection of permutations is shown for brevity. Whereas the challenge only displayed a single activity with its in-links and out-links 

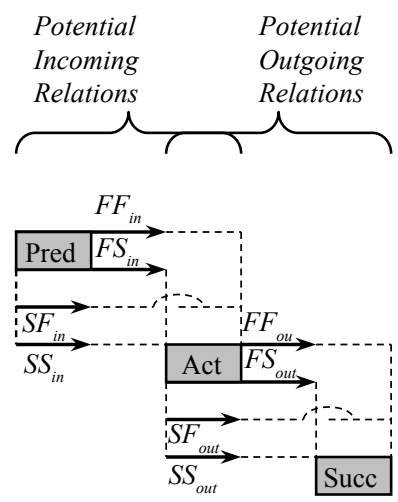

Fig. 1: Activity triplet with relations.

(Hajdu et al. 2016) without distinguishing or showing how exactly they attach to the predecessor and successor, this paper examines the full triplet of activities with explicit link types as shown in Figure 1 with 15 permutations for the links in each of its two pairs, which gives a total of $15 \times 15=225$ permutations. Following the proof, nonlinear activities are reviewed regarding criticality and float by using linear schedules that show work over time, nonlinearity is discretized with multiple segments, buffers replace lags as discrete end-point links become continuous relations, and their constellations are examined as to their behavior. The scope of this paper is limited to minimum constraints; future research will explore how maximum constraints, which Hajdu (1997) studied for networks and Reis and Lucko (2016) for linear schedules, cause criticality configurations.

\section{Research question 1: analysis of criticality constellations}

\subsection{Computer simulation of all permutations}

The aforementioned 225 permutations have been simulated in a computer implementation. The activity sequence is predecessor (pred), current activity (act), and successor (succ). Each activity is connected with at least one other activity (Figure 1), and the coding ensures that the current activity is never "skipped" by only connecting its predecessor and successor, which would preclude calculating a valid start and finish. Such "bypassing" links that could connect the predecessor directly with the successor are technically conceivable but are excluded here for brevity and may be examined in future research. Formulas for incoming (in) and outgoing (out) links of a current activity have been implemented in a forward pass calculation. Randomized variables are integer durations of the three activities within a set range and lags of all in-links and all out-links. If in-links and out-links (n.b. they are minimum constraints) give different tentative start or finish dates, the maximum value is used to maintain the integrity of all non-interruptible activity durations as start plus duration equals finish $(\mathrm{S}+\mathrm{D}=\mathrm{F})$. Outputs are counts of the randomized three-activity schedules as to whether they incur $\{+-\},\{-+\},\{00\}$, $\{+0\},\{0+\},\{++\},\{-0\},\{0-\}$, or $\{--\}$ behavior of the project if the activity duration increases or decreases (both of which are examined in parallel). Comparing the project finish, i.e., successor finish $F_{\text {suce }}$, between unchanged and increased or unchanged and decreased current activity duration gives the desired counts. An actual result of the simulation is, e.g., $96+8+75+30+14+2+0+0+0=$ 225 (in the order of listed behaviors). The cases of $\{+-\}\{00\}$ are unsurprisingly observed most often. Three hypothetical cases $\{-0\},\{0-\}$, and $\{--\}$ are marked to give an alert if incurred. Both simulation runs were executed for several thousand instances. None of them ever generated any $\{-0\}$, $\{0-\}$, or $\{--\}$ behavior. The simulation implies that these three cases are either very rare or do not exist. While it is not validation by itself, this finding informs the following mathematical analysis of criticality constellations to gain a proof for or against their possible existence.

\subsection{Proof by contradiction}

If any activity pair is connected by more multiple different link types, unless their lags happen to give exactly the same start and finish of the successor, one of them will typically dominate and can be treated like a single FS, SS, FF, or SF link or the common SS-FF combination that synchronizes the extent of their concurrent performance. In fact, schedulers would consider connecting activity pairs with multiple link types to be redundant and superfluous. Therefore, while lags can clearly greatly affect the simulation results, it suffices to examine only dominant behavior. As mentioned, mathematically this is represented as $\mathrm{S}_{\mathrm{act}}=\max \{\mathrm{F}$ pred $\left.+\mathrm{FS}_{\text {lag, }}, \mathrm{F}_{\text {pred }}+\mathrm{FF}_{\text {lag }}-\mathrm{D}_{\text {act, }}, \mathrm{S}_{\text {pred }}+\mathrm{SF}_{\text {lag }}-\mathrm{D}_{\text {act }}, \mathrm{S}_{\text {pred }}+\mathrm{SS}_{\text {lag }}\right\}$.

The triplet of activities is examined in two pairs regarding their constellation and behavior. The normal, neutral, and reverse basic cases of Figure 2 prove in and of themselves that the $\{+-\},\{-+\}$, and $\{00\}$ behaviors are possible. The cases of Figure 2 prove that $\{+0\},\{0+\}$, and $\{++\}$ exist. Combining the desired properties gives the following:

- $\{0-\}$ : on the one hand, the neutral property 0 ? means that a longer activity causes the project duration to stay the same (? is a placeholder for shorter activity). This can only occur if the activity is held at one end and the other 


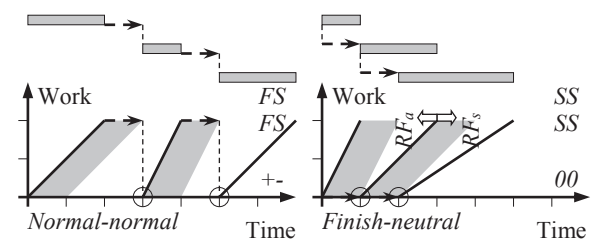

(a)

(c)

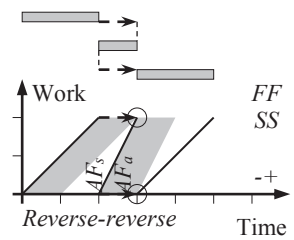

(e)

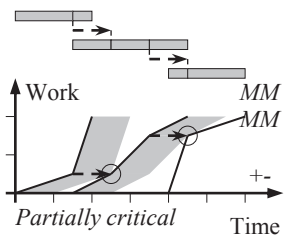

(g)

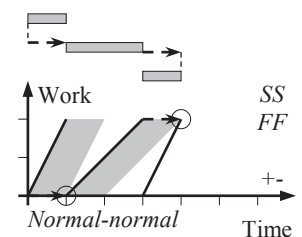

(b)

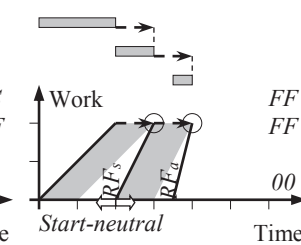

(d)

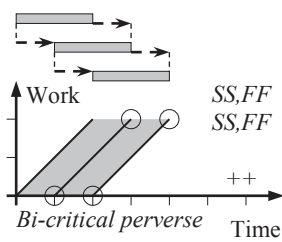

(f)

Fig. 2: (a) Normal by FS-FS; (b) normal by SS-FF; (c) finish-neutral by SS-SS; (d) start-neutral by FF-FF; (e) reverse by FF-SS; (f) perverse by SS, FF-SS, and FF; (g) partially critical by MM-MM.

Note: Some essentially equivalent cases are omitted here for brevity.

end can rotate (earlier if finish is held or later if start is held as shown in Figure 2c and d). Predecessor and successor links must effectively bypass the current activity. On the other hand, the normal property ?- means that a shorter activity causes a shorter project. This can only occur (for end-point links) if the predecessor holds the current activity at its start, which is held at its finish by the successor (in analogy to the property placeholder, these requirements can be represented as ?S-S? or ?F-F? for the neutral property and ?S-F? for the normal property, where the two activity pairs are separated by a hyphen). These two requirements contradict; $\{0-\}$ is impossible.

- $\{-0\}$ : on the one hand, the reverse property -? means that a longer activity causes a shorter project. This can only occur (for end-point links) if the predecessor holds the current activity with a ?F and the successor holds it with a S?. This reverses the criticality flow through it. It must start earlier to be longer and pull its successor earlier as well. On the other hand, the neutral property ?0 means that a shorter activity does not impact the project duration. Thus, one free end of the current activity can rotate as shown in Figure $2 \mathrm{c}$ and d, while its other end is held by either ?S-S? or ?F-F? as links from the predecessor and to the successor. These requirements contradict; $\{-0\}$ is impossible.

- $\{--\}$ : the same reasoning as for the previous two cases applies. To summarize, the - ? property requires a "hinge" constellation of ?F-S? among the triplet. However, the ?- property requires a free end to rotate (?S-S? or ?F-F?). These requirements contradict. The behavior $\{--\}$ is impossible. Therefore, the three hypothesized cases that combine these properties cannot exist, which matches with the randomly generated output of the computer simulation.
The special case of "perverse" behavior shown in Figure $2 \mathrm{f}$ merits attention. Different than the other basic cases, it has two links between each activity pair. These let it switch the dominant link pair from increased to decreased current activity duration. This gives the interesting but practically undesirable effect that project duration always increases.

\subsection{Role of start-to-finish relation}

The start-to-finish relation, which almost, but not quite inverts the logic of the activity pair, is rare among FS, SS, $\mathrm{FF}$, and SF in practice, and actual examples have proven difficult to find in the authors' experience. A real-world case where this is appropriate is, e.g., installing a prefabricated stair in an atrium, where the start of its installation triggers the finish of the façade panels, in which an opening has been left to lift and move the stair into the building. Since it is noticeably absent from the basic cases of Figure 2, it is merited to explore its role. If predecessor and successor are assumed to not change (as they are here), then some basic cases can be created using SF and lag with the same start and finish points but would have changed criticality. Figure 2e could use FF-SF, SF-SS, or SF-SF, whose lags would be one or two activity durations, but the predecessor or successor would become noncritical. In Figure 2c and d, if the second (first) link could be replaced by SF, then the successor (predecessor) would become noncritical. However, SF cannot create other cases (Figure 2a and b) whose behavior hinges on a normal direction of the criticality flow through the current activity. To explain the special role of SF, note that FS traverses both activities, SS and FF one each (in successor or predecessor only), but SF circumvents both, which gives it a special 
role of preventing any criticality constellations. This would even allow a triplet of start/finish-neutral activities with either SF-FF or SS-SF, which (if understanding criticality that a duration change will impact the project finish and assuming that a duration change also moves the "free" activity end while holding the other fixed) would mean that all three activities act as noncritical.

\section{Research question 2: analysis of nonlinear progress}

\subsection{Extension to linear schedules}

To represent nonlinear activities, it becomes necessary to use linear schedules, because they show the changing productivity as positive or negative curvature or straight lines, which can be interpreted as learning, fatigue, or fixed progress. End-point-link lags of network schedules become continuous buffers, which are represented as gray shaded areas in linear schedules. Note that they can be quantified either in time or work units, which gives a different shape at buffer ends (Lucko and Peña Orozco 2009). This paper treats time buffers. Lucko (2008, p. 8) showed a "range relationship" as the limit of increasingly many end-point links between two activities with linear progress and demonstrated (Lucko 2009) how with activity productivities, buffers, and sequence as input, the SS or FF links automatically emerge as output of a two-dimensional (2D) (work and time) linear schedule. However, a one-dimensional (time only) network schedule requires specific link types as input for the critical path method or precedence diagramming method, which requires a priori decisions by the scheduler and hampers using concurrent activities (Allen 1983) that could minimize project duration (Hajdu 1997).

\subsection{Partial criticality}

Harmelink (2001) demonstrated that any continuous monotonously progressing activity in a linear schedule can be partially critical and that such partial criticality can create no more than three different segments from its start to its finish: start-noncritical, critical, and finishnoncritical. Further analysis reveals special cases where one or multiple segments may have a length of zero. All activities, including nonlinear ones, can be reduced to such cases. For example, activities in Figure 2a have noncritical segments of zero length, i.e., they contain only a critical segment, but the current activity in Figure $2 \mathrm{c}$ and $\mathrm{d}$ shows a finish-noncritical and start-noncritical segment. Noncritical segments can be understood as free ends of an activity that can rotate and consume rate float (RF) (Awwad and Ioannou 2007) by starting earlier (accelerate, $\mathrm{RF}_{\mathrm{a}}$ ) or finishing later (slowdown, $\mathrm{RF}_{\mathrm{s}}$ ), while the middle critical segment is held from both ends. Note that starting earlier proactively may be somewhat unrealistic in practice, as delays are not usually anticipated.

If activities are discretized with straight-line segments, this means that activity constellations with at most three segments of different productivities would need to be studied. For monotonous curvature as the conjecture assumes, the critical segment in the middle is reduced to a single point of contact. As Figure $2 \mathrm{~g}$ shows for discretized activity segments of different productivity, this means that an activity pair with one or both nonlinear activities can only connect at their start or finish to form an end-point SS or FF or a mid-point pair for a mid-point relation (MM). Like SS and FF, this relation automatically emerges in linear schedules with nonlinear progress from the relative slopes and curvature of an activity pair, whereas the aforementioned various studies on network extensions had to define it deliberately as a generalized mid-point link without realizing its important role. The critical segment of the current activity has a normal-normal impact on the project duration; the two noncritical segments have RF.

\subsection{Float in linear schedules}

Float in linear schedules that is consumed by rotation of an activity segment has been called (productivity) RF to indicate that the longer or shorter duration is created by a lower or higher productivity. It is measured in work units divided by time units, and the value at the activity start and finish is equal to the free float of said activity in time units. Figure $2 c$ and $d$ shows that $R F$ is limited by the extent of the predecessor buffer (RFa) or successor activity (RFs). Consuming more than them would change the criticality and thus is beyond the scope of this paper.

For the criticality analysis, this paper establishes a new float type, whose definition may appear counterintuitive. This new concept is based on the fact that critical segments can change productivity and duration in practice. Same as for RF, the edge length of AF in time units is the increase or decrease in duration before criticality changes.

- Apparent float is defined as RF by which a critical activity segment in a linear schedule could be delayed but start earlier $\left(\mathrm{AF}_{\mathrm{s}}\right)$ or shortened but finish later $\left(\mathrm{AF}_{\mathrm{a}}\right)$ without becoming noncritical, as Figure 2e illustrates. Its rotational value is limited by the predecessor buffer $\left(\mathrm{AF}_{\mathrm{s}}\right)$ and the slope of the current activity itself $\left(\mathrm{AF}_{\mathrm{a}}\right)$. 


\subsection{Nonlinear activity progress}

Assuming that nonlinear activity progress exhibits a monotonous behavior, an activity could have an increase, decrease, or no change in productivity in the work-time diagram of a linear schedule. In analogy to the basic cases, further pairs and triplets will be studied in regard to criticality constellations. Concave or convex shapes with a single positive or negative curvature can be compared to adjacent activities by whether their average slope is higher or lower, as shown by the dashed lines in Figure 3. They can thus be reduced to a known basic case that forms either SS, FF, or both SS and FF relations. Thus, the diverging Figure $3 \mathrm{a}$ and $\mathrm{b}$ are equivalent, as are the converging Figure $3 \mathrm{c}$ and $\mathrm{d}$. Parallel (or balanced) progress exists if the average activity productivity and its buffers are equal for an activity pair; Figure 3e demonstrates that this is possible for \{negative-zero\}, \{zero-positive\}, and \{negative-positive\} curvature pairs that bend "toward each other." Figure $3 \mathrm{f}$ shows that for \{positive-negative\} pair, it is impossible; it will instead form an MM relation (IBM 1964). The number of permutations of hhigh, low, equal\} slopes is given by selecting two of four nonlinear triplet links with replacement, which is calculated as $42=16$ criticality configurations, which are listed as follows: $\{\mathrm{SS}-\mathrm{SS}\},\{\mathrm{SS}-\mathrm{FF}\},\{\mathrm{FF}-\mathrm{FF}\},\{\mathrm{FF}-\mathrm{SS}\},\{\mathrm{SS}-(\mathrm{SS}, \mathrm{FF})\}$, $\{\mathrm{FF}-(\mathrm{SS}, \mathrm{FF})\},\{(\mathrm{SS}, \mathrm{FF})-\mathrm{SS}\},\{(\mathrm{SS}, \mathrm{FF})-\mathrm{FF}\},\{(\mathrm{SS}, \mathrm{FF})-(\mathrm{SS}, \mathrm{FF})\}$, \{SS-MM $\}, \quad\{$ FF-MM $\}, \quad\{$ MM-SS $\}, \quad\{$ MM-FF $\}, \quad\{$ MM-MM $\}$, $\{(\mathrm{SS}, \mathrm{FF})-\mathrm{MM}\}$, and $\{\mathrm{MM}-(\mathrm{SS}, \mathrm{FF})\}$.

Allowing multiple curvatures within nonlinear activities (shown as S-curves in Figure 3) increases the number of criticality constellations, because an activity pair may now develop not just point-to-point MM links as shown in Figure $3 \mathrm{~g}$ but may align in any or all of their three partial criticality segments as shown in Figure 3f, which has a continuous relation from SS to MM. A constant buffer is assumed here. The observation of Harmelink (2001) on (start-noncritical, critical, finish-noncritical) partial criticality segments remains valid, and each of these three segments can have a zero or nonzero length, so that it may be more appropriate to label the activity pair by which of these three segments are related. Permutations are now selected from SS, FF, MM, (SS,FF), plus new aligned segment relations of start-noncritical, critical, and finish-noncritical (SN, CC, and FN) for a maximum two of seven with replacement, which is $7^{2}=49$.

\section{Research question 3: generalization of influence lines}

Influence lines have been used to graphically represent how changes in the duration of a single activity impact the project duration (Hajdu et al. 2016). However, it is simplistic and inaccurate to assume that the only determining factor for project duration is just the activity duration itself. Rather, its interaction with its predecessors and successors constitutes a three-way sensitivity analysis. Figure 2 has illustrated the possible constellations of three relative durations. Yet, three activity durations plus one project duration cannot be represented in a single three-dimensional (3D) diagram, so that each diagram is a "snapshot" for a specific activity duration between its predecessor and its successor. An animation of several such diagrams can show the dynamic interaction of a sequence of three activities vis-à-vis the dependent variable project duration. Figure $4 a$ and $b$ shows such snapshots for 1 day and 4 days duration of the activity, respectively, assuming the same time buffer of 1 day as in the previous examples. It is proposed to call these new diagrams influence surfaces. They resemble the constellations of Figure $2 \mathrm{~b}$ and e but additionally show the effect of varying both the predecessor and successor durations. Reading them is straightforward, as distinct zones emerge. For example, in Figure $4 \mathrm{~b}$, the horizontal plateau zone indicates that the

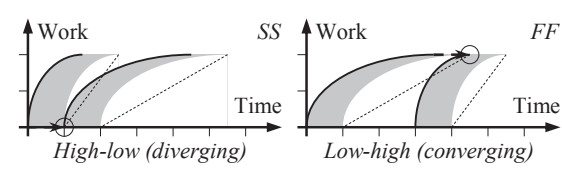

(a)

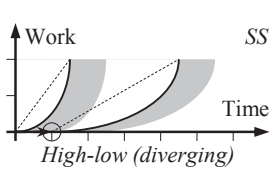

(b) (c)

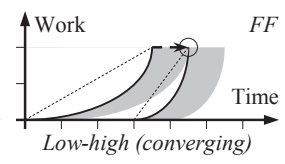

(d)

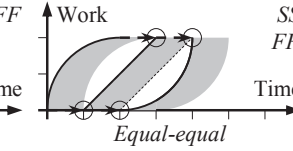

(e)

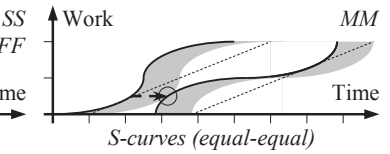

(g)

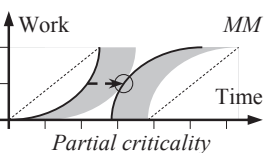

(f)

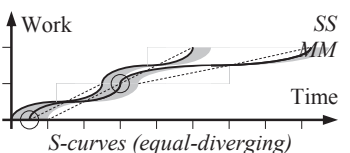

(h)

Fig. 3: Relative productivities of nonlinear curvatures. (a) Negative diverging; (b) positive diverging; (c) negative converging; (d) positive converging; (e) negative-zero-positive equal-equal; (f) positive-negative partial criticality; (g) S-curves equal-equal; and (h) S-curves equal-diverging. 

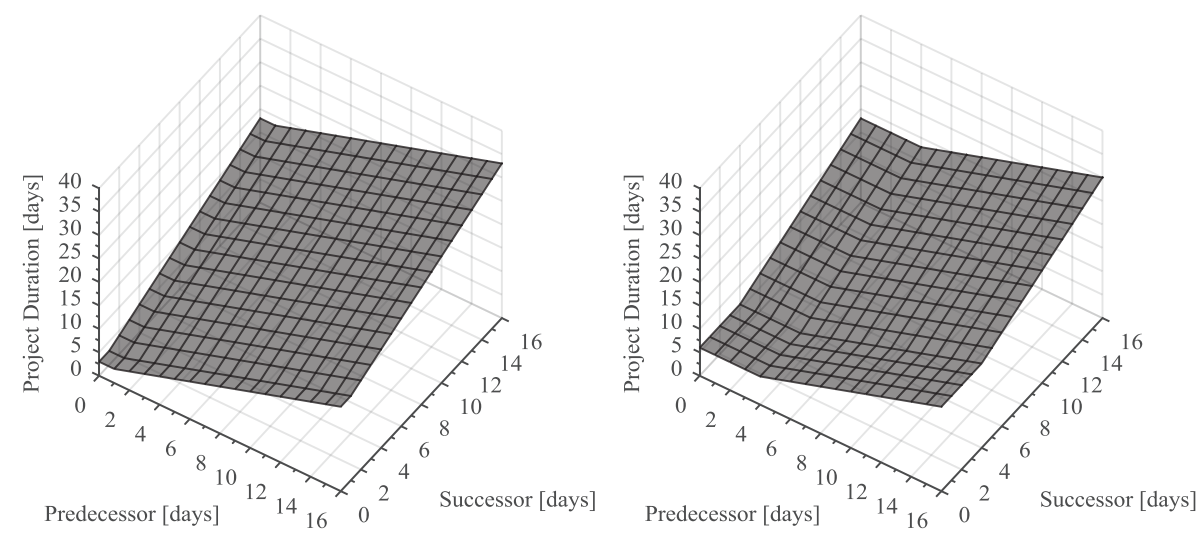

(a)

(b)
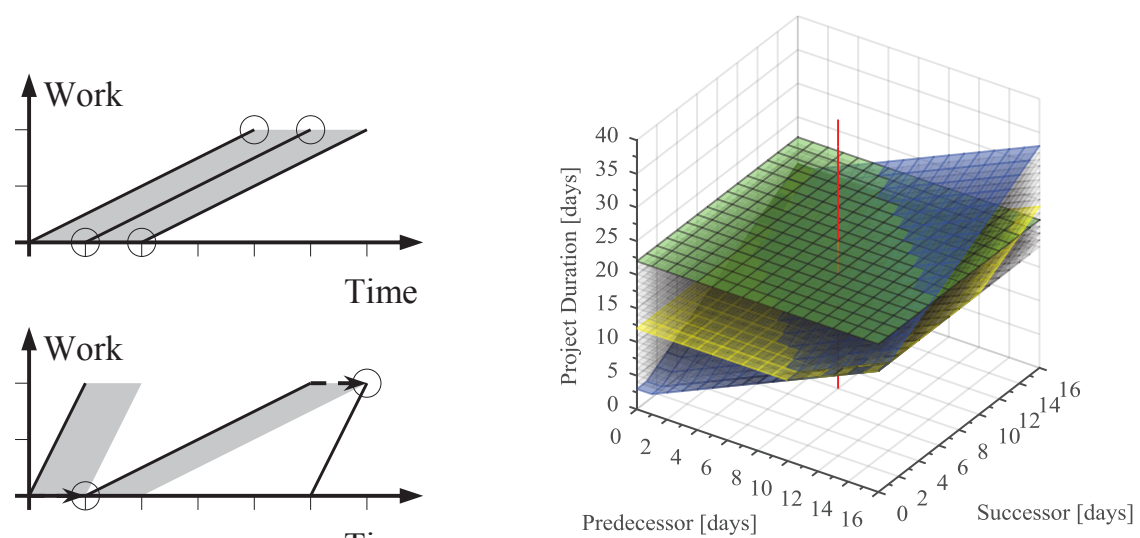

(c)

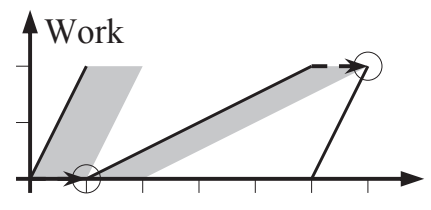

Time

(d)

Fig. 4: Project duration for activity while varying predecessor and successor. (a) Activity duration = 1 day; (b) activity duration = 4 days; (c) predecessor and successor duration $=1$ day -4 days; and (d) combined influence surfaces for activity duration $=1$ day-16 days.

project duration remains fixed at 6 days for both the predecessor and successor taking between 0 day and 4 days. Figure 4c illustrates two points on the influence surface as linear schedules, here with the predecessor and successor from Figure $4 \mathrm{~b}$ at 1 day (because a zero duration would be unrealistic) and 4 days. Note that these predecessor and successor ranges coincide with the newly defined apparent float of the activity, except that apparent float keeps criticality unchanged while allowing the project duration to vary, but the influence zone here has a fixed project duration for varying predecessor and successor durations. The remaining three influence zones - sloped areas in the diagram - are explained as follows: If either the predecessor or successor grows their duration above 4 days, then the project duration grows proportionally. If both increase together, then the project duration grows more due to their combined effect. Each zone represents how changes in activities will impact the project.

criticality constellations can be extracted from considering multiple influence surfaces (i.e., an influence solid whose dynamic layers are said as influence surfaces).
Figure $4 \mathrm{~d}$ shows several of these activity durations in blue, yellow, and green (1 day, 8 days, and 16 days, respectively). The point where a vertical red line in Figure $4 \mathrm{~d}$ for predecessor-successor combinations crosses each influence surface gives project durations, which when plotted over the activity duration of each layer gives exactly the aforementioned 2D influence line (Hajdu et al. 2016).

Combining the individual snapshots in the same diagram is possible but becomes harder to read. Figure $4 \mathrm{~d}$ shows such a dynamic set of snapshots for the activity as stacked layers. Varying only the activity duration but not its predecessor or successor is equivalent to a vertical cut as the red line in Figure $4 \mathrm{~d}$ indicates. Viewing it as projections from the axis planes shows the two-way interaction of just varying predecessor or successor for different activity durations (i.e., layers).

This approach can theoretically be generalized toward multiple predecessors on one axis and multiple successors on another axis. The dominant ones will become visible in the 3D influence surface, and nondominant ones may be omitted. This may give a more faceted influence surface. 


\section{Contributions and recommendations}

The contribution of this paper to the body of knowledge is threefold: First, a proof has been provided that no more than six known criticality constellations can exist for activities with linear progress that are linked only by end-point relations. The rare SF has been found to counteract the formation of criticality constellations, because it bypasses activities. Second, more intricate criticality constellations exist for nonlinear continuous activities, because of their unique ability to induce mid-point relations and partial criticality in any activity pair with unequal curvature as shown in Figure 3f. This is also true for piecewise linearly segmented activities as shown in Figure 2g, which generate similar constellations, because their segments are bounded by slope changes. These findings do not contradict the conjecture, which had allowed "inner" points (Hajdu et al. 2016), i.e., mid-point relations, in referring to a previously published generalization (Bokor and Hajdu 2015; Hajdu 2015c). Third, a generalization of influence lines toward influence surfaces presents a detailed visualization of how three activities can dynamically interact.

Categorizing their criticality constellations by their dominant link behavior is beyond the scope of this paper. If multiple different link types (point-to-point and continuous) connect an activity pair, their criticality constellation could be classified by their dominant link but is hypothesized to not introduce any new types. If nonlinear time and work buffers are allowed (as opposed to only nonlinear activities), they could then be superimposed onto the relative productivities of their host activity pairs and treated per their combined slopes in analogy to the known cases but are hypothesized to not introduce any new types. It is recommended to study these two new conjectures under future research. However, more constellations could arise if multiple crews are introduced as productive resources that alternate across individual work units, and individual workflows may be interruptible, which should be studied in more detail.

Further research is recommended to generalize this end-point and continuous analysis to links that connect points anywhere on activities (Hajdu 2015c) and also to consider both minimum and maximum constraints as lags on links (Hajdu 1997) for criticality, as well as their extension in linear schedules, where maximum constraints have just been introduced (Reis and Lucko 2016). The effects of partially critical activity segments on project duration should also be categorized.

\section{Acknowledgments}

The creators of the inaugural Creative Construction Conference challenge are thanked for the interesting puzzle. The authors thank two anonymous reviewers for constructive criticism, which has improved the clarity of this paper. This paper is dedicated to the memory of Mr. James E. Kelley, Jr, with whom the first author corresponded in 2007.

The support of the National Science Foundation (Grant CMMI-1265989) for portions of the work presented here is gratefully acknowledged. Any opinions, findings, and conclusions or recommendations expressed in this material are those of the authors and do not necessarily represent the views of the National Science Foundation.

\section{Disclosure}

This paper is an expanded version of an earlier manuscript by the same authors that was printed in Procedia Engineering proceedings of the 2017 Creative Construction Conference.

\section{References}

Allen, J. F. (1983). Maintaining knowledge about temporal intervals. Communications of ACM, 26(11), pp. 832-843.

Awwad, R. E., \& loannou, P. G. (2007). Floats in RSM: Repetitive scheduling method. In: Proceedings of the Construction Research Congress, Grand Bahama Island, The Commonwealth of the Bahamas, May 6-8, 2007, American Society of Civil Engineers, Reston, VA, pp. 1133-1140.

Bokor, O., \& Hajdu, M. (2015). Investigation of critical activities in a network with point-to-point relations. Procedia Engineering, 123, pp. 198-207.

Crandall, K. C. (1973). Project planning with precedence lead/lag factors. Project Management Quarterly, 4(3), PMI-18-PMI-27.

Fazar, W. (1962). The origin of PERT. The Controller, 1962(December), pp. 598-621.

Fondahl, J. W. (1962). A Non-Computer Approach to the Critical Path Method for the Construction Industry, 2nd ed., November 1961, Revised 1962, Technical Report No. 9, Prepared under research contract NBy-17798, Bureau of Yards and Docks, U.S. Navy, Distributed by The Construction Institute, Stanford University, Stanford, CA, $133 \mathrm{pp}$.

Francis, A. (2004). La modélisation chronographique de la planification des projets de construction. Dissertation, École de Technologie Supérieure, Montréal, QC, Canada, 331 pp.

Hajdu, M. (1997). Network Scheduling Techniques for Construction Project Management. Kluwer Academic, Dordrecht, The Netherlands. 
Hajdu, M. (2015a). Continuous precedence relations for better modelling overlapping activities. Procedia Engineering, 123, pp. 216-223.

Hajdu, M. (2015b). History and some latest developments of precedence diagramming method. Organization, Technology and Management in Construction, 7(2), pp. 1302-1314.

Hajdu, M. (2015c). One relation to rule them all: The point-to-point precedence relation that substitutes the existing ones. In: Proceedings of the CSCE International Construction Specialty Conference, Vancouver, BC, Canada, June 8-10, 2015, Canadian Society for Civil Engineering, Montréal, QC, Canada, pp. 827-837.

Hajdu, M. (2016). How many types of critical activities exist? A conjecture that needs a proof. In: Presentation, Creative Construction Conference, Budapest, Hungary, June 25-28, 2016, Diamond Congress, Budapest, Hungary.

Hajdu, M., Lucko, G., \& Su, Y. (2017). Singularity functions for continuous precedence relations and nonlinear activitytime-production functions. Automation in Construction, 79(July), pp. 31-38.

Hajdu, M., Skibniewski, M. J., Vanhoucke, M., Horvath, A., \& Brilakis, I. (2016). How many types of critical activities exist? A conjecture that needs a proof." Procedia Engineering, 164, pp. 3-11.

Harmelink, D. J. (2001). Linear scheduling model: Float characteristics. Journal of Construction Engineering and Management, 127(4), pp. 255-260.

Harris, R. B. (1978). Precedence and Arrow Networking Techniques for Construction. John Wiley and Sons, New York City, NY.

IBM. (1964). User's Manual for IBM 1440 Project Control System (PCS). International Business Machines, Armonk, NY.

Kallantzis, A., \& Lambropoulos, S. (2004). Critical path determination by incorporation minimum and maximum time and distance constraints into linear scheduling. Engineering, Construction and Architectural Management, 11(3), pp. 211-222.

Kelley, J. W., \& Walker, M. R. (1959). Critical path planning and scheduling. In: Proceedings of the Eastern Joint Computer Conference, Boston, MA, December 1-3, 1959, National Joint Computer Committee, Association for Computing Machinery, New York City, NY, 16, pp. 160-173.

Kelley, J. W., \& Walker, M. R. (1989). The origins of CPM: A personal history. pmNetwork, 3(2), pp. 7-22.

Kim, S.-Y. (2012). CPM schedule summarizing function of the beeline diagramming method. Journal of Asian Architecture and Building Engineering, 11(2), pp. 367-374.
Lucko, G. (2008). Analysis of linear schedules with singularity functions versus critical path method. In: Presented at PMI College of Scheduling Annual Conference, Chicago, IL, May 4-7, 2008, Project Management Institute, Newtown Square, PA, $12 \mathrm{pp}$.

Lucko, G. (2009). Productivity scheduling method: Linear schedule analysis with singularity functions. Journal of Construction Engineering and Management, 135(4), pp. 246-253.

Lucko, G., \& Gattei, G. (2016). Line-of-balance against linear scheduling: Critical comparison. Proceedings of the Institution of Civil Engineers - Management, Procurement and Law, 169(1), pp. 26-44.

Lucko, G., \& Peña Orozco, A. A. (2009). Float types in linear schedule analysis with singularity functions. Journal of Construction Engineering and Management, 135(5), pp. 368-377

Mauchly, J. W. (1962). Critical-path scheduling. Chemical Engineering, April 16, pp. 139-154.

Mubarak, S. A. (2015). Construction Project Scheduling and Control, 3rd edn. Pearson/Prentice-Hall, Upper Saddle River, NJ.

Plotnick, F. L. (2006). RDM - relationship diagramming method. In: Transactions of the AACE Annual Meeting, Last Vegas, NV, June 19-22, 2006, Association for the Advancement of Cost Engineering International, Morgantown, WV, PS.08.1PS.08.10.

Ponce de Leon, G. (2008). Graphical planning method (A new network-based planning/scheduling paradigm). In: Presented at PMI College of Scheduling Annual Conference, Chicago, IL, May 4-7, 2008, Project Management Institute, Newtown Square, PA, $10 \mathrm{pp}$.

Reis, J. S., \& Lucko, G. (2016). Productivity scheduling method with maximum constraints. International Journal of Construction Management, 16(1), pp. 77-93.

Rösch, W. (1970). Roy-Typ und Fondahl-Typ: Ein Beitrag zur Frage der Tätigkeitsgraphen in Bauwesen. Dissertation, Technical University Carolo-Wilhelmina of Brunswick, Brunswick, Germany, 144 pp.

Roy, G. B. (1959). Contribution de la théorie des graphes á l'étude de certains problémes linéaries. Comptes Rendus de Séance de l'Académie des Sciences, 248(April 27, 1959), pp. 2437-2439.

Wiest, J. D. (1981). Precedence diagramming method: Some unusual characteristics and their implications for project managers. Journal of Operations Management, 1(3), pp. 121-130. 